TUM-HEP 809/11

\title{
Understanding neutrino properties from decoupling right-handed neutrinos and extra Higgs doublets
}

\author{
Alejandro Ibarra and Cristoforo Simonetto \\ Physik-Department T30d, Technische Universität München, \\ James-Franck-Straße, 85748 Garching, Germany
}

\begin{abstract}
Low energy effects induced by heavy extra degrees of freedom are suppressed by powers of the large mass scale, thus preserving, if sufficiently heavy, the successes of the Standard Model in describing low energy phenomena. However, as is well known, heavy right-handed neutrinos may play an important role in low energy phenomenology as an explanation of the smallness of neutrino masses. We consider in this paper an extension of the Standard Model by heavy right-handed neutrinos and heavy Higgs doublets and we show, using a renormalization group approach, that this model can simultaneously provide an explanation for the small neutrino masses and for the mild hierarchy observed between the atmospheric and the solar mass splittings, even when the extra degrees of freedom are very heavy. We analyze the necessary conditions to reproduce the oscillation parameters and we discuss possible experimental signatures of this model.
\end{abstract}

\section{Introduction}

The last fifteen years have witnessed a tremendous experimental progress in neutrino physics, leading to a good determination of two mass splittings, two mixing angles and a fairly stringent bound on the third angle [1]. The most conspicuous difference between quark and neutrino properties is without any doubt the smallness of neutrino masses. Furthermore, the better and better measurements of neutrino parameters have also revealed the existence of large mixing angles in the leptonic sector and the existence of a relatively mild mass hierarchy between the two heaviest neutrino masses [2].

Extending the particle content of the Standard Model (SM) with three heavy righthanded neutrinos, thus implementing the (type I) see-saw mechanism [3], solves very elegantly the problem of generating small neutrino masses and opens new opportunities to understand the puzzles of the existence of large mixing angles and a small mass hierarchy. Unfortunately, whereas the seesaw mechanism does not make any generic prediction 
about the leptonic mixing matrix, it tends to predict a neutrino mass hierarchy which is much larger than the one inferred from experiments [4]. Namely, under the plausible assumption that the neutrino Yukawa couplings have hierarchical eigenvalues, as observed in the quark and the charged lepton sectors, the mild mass hierarchy observed between the solar and the atmospheric mass splitting can only be accommodated in very special cases. One possibility arises when the hierarchies between the masses of the heavy right-handed neutrinos is much larger than the hierarchy between the neutrino Yukawa eigenvalues, in which case the right-handed mixing angles have to be tiny. Alternatively, when the hierarchy in the masses of the heavy right-handed neutrinos is comparable to the hierarchy in the Yukawa couplings, it is possible to accommodate the observed mild neutrino mass hierarchy only for certain, possibly fine-tuned, choices of the right-handed mixing angles.

Further extensions of the type I see-saw model have been considered in the literature. A minimal possibility consists on introducing one extra Higgs doublet, which leads to radiatively induced neutrino masses. Most works impose an additional $Z_{2}$ symmetry, under which the Standard Model particles are even, whereas the right-handed neutrinos and the extra Higgs doublet are odd [5, 6]. With this assignment, the tree level neutrino mass vanishes and the leading contribution is hence the radiatively generated one. In this scenario, the right-handed neutrinos and the extra Higgses could be directly produced at colliders while being the neutrino masses in the measured range, thanks to the loop suppression and an appropriate choice of the neutrino Yukawa and Higgs self-couplings. Furthermore, the $Z_{2}$ symmetry ensures the absence of tree level flavour changing neutral currents. On the other hand, it has also been discussed the general two Higgs doublet model (2HDM), without imposing ad-hoc discrete symmetries [7]. In this case, both mechanisms of neutrino mass generation are present, naturally leading to a mild neutrino mass hierarchy [8].

In this paper we carefully analyze the mechanism of neutrino mass generation in the two Higgs doublet model extended with right-handed neutrinos. Rather than being motivated by finding signatures of new physics in experiments at the energy frontier or at the intensity frontier, we are motivated by constructing a simple and natural framework capable to explain the observed neutrino parameters while preserving the successes of the Standard Model. This approach is inspired in the high scale see-saw mechanism, which despite its well known lack of testability, still stands as the most compelling explanation for the small neutrino masses.

We will argue that by making all the new particles heavy it is possible to simultaneously explain the smallness of neutrino masses and the mildness of the neutrino mass hierarchy, without jeopardizing any of the successes of the Standard Model. Remarkably, in this model only one right-handed neutrino suffices to generate two neutrino mass scales: the atmospheric neutrino mass scale will be generated at tree level, whereas the solar mass scale will be generated by the radiative corrections to the effective neutrino mass matrix. Both neutrino masses are suppressed by the large right-handed neutrino mass scale, thus explaining the tininess of neutrino masses. On the other hand, the ratio of the two mass scales is suppressed by the loop factor and enhanced by a large logarithm 
of the ratio of the heavy right-handed neutrino mass to the Higgs mass, resulting in a neutrino mass ratio which can be roughly of the correct size. Finally, the decoupling of the extra Higgs degrees of freedom ensures the absence of large contributions to the flavour and CP violating processes, both in the leptonic sector and in the quark sector, as well as to the electroweak precision measurements.

In Section 2 we review the basic features of the two Higgs doublet model, the various problems which arise in this very minimal extension of the Standard Model, and how they can be circumvented altogether by decoupling the extra scalar degrees of freedom. In Section 3 we show, using a renormalization group approach, that even in the decoupling limit the extra Higgs particles can play an important role in low energy neutrino physics, as an explanation for the mild hierarchy observed between the atmospheric and the solar neutrino mass scales. The viability of this model requires at least one righthanded neutrino and two Higgs doublets; in Section 4 we comment on the differences of this framework with another minimal framework of neutrino masses, namely the two right-handed neutrino model with just one Higgs doublet. In Section 5 we calculate the corrections to the leptonic mixing matrix induced by quantum effects, and we argue that a non-zero $\theta_{13}$ is generically expected, as well as a a deviation from the maximal atmospheric angle which is correlated to the angle $\theta_{13}$. Lastly, in Section 7 we present our conclusions.

\section{Benefits of the decoupling limit of the 2HDM}

We consider an extension of the SM consisting on adding to the particle content one additional Higgs doublet, with identical quantum numbers as the SM Higgs doublet. The most general Higgs potential reads [9, 10]:

$$
\begin{aligned}
V & =m_{11}^{2} \Phi_{1}^{\dagger} \Phi_{1}+m_{22}^{2} \Phi_{2}^{\dagger} \Phi_{2}-\left[m_{12}^{2} \Phi_{1}^{\dagger} \Phi_{2}+\text { h.c. }\right] \\
& +\frac{1}{2} \lambda_{1}\left(\Phi_{1}^{\dagger} \Phi_{1}\right)^{2}+\frac{1}{2} \lambda_{2}\left(\Phi_{2}^{\dagger} \Phi_{2}\right)^{2}+\lambda_{3}\left(\Phi_{1}^{\dagger} \Phi_{1}\right)\left(\Phi_{2}^{\dagger} \Phi_{2}\right)+\lambda_{4}\left(\Phi_{1}^{\dagger} \Phi_{2}\right)\left(\Phi_{2}^{\dagger} \Phi_{1}\right) \\
& +\left[\frac{1}{2} \lambda_{5}\left(\Phi_{1}^{\dagger} \Phi_{2}\right)^{2}+\lambda_{6}\left(\Phi_{1}^{\dagger} \Phi_{1}\right)\left(\Phi_{1}^{\dagger} \Phi_{2}\right)+\lambda_{7}\left(\Phi_{2}^{\dagger} \Phi_{2}\right)\left(\Phi_{1}^{\dagger} \Phi_{2}\right)+\text { h.c. }\right] .
\end{aligned}
$$

Despite being such a simple extension of the Standard Model, the introduction of a second Higgs doublet in general jeopardizes many of the successes of the Standard Model. More concretely, the Higgs potential has now a richer structure including electrically charged directions, which may lead to the spontaneous breaking of the electromagnetic $U(1)$ symmetry if there are minima along those directions. Besides, the extra Higgs doublet contributes to the oblique parameters $S, T$ and $U$ [11], possibly leading to values in conflict with electroweak precision data. Lastly, the new, in general flavour violating, couplings of the fermions to the extra Higgs doublet could lead to too large flavour changing neutral currents or lepton flavour violation.

It is interesting that all these problems are simultaneously solved in the decoupling limit of the two Higgs doublet model, which is defined as the limit where one of the 
Higgses is kept light, with a mass comparable to the Z-boson mass, while the rest acquire masses much larger than $M_{Z}$.

To show the absence of charge breaking minima in the decoupling limit we will work without loss of generality in the Higgs basis where $m_{12}=0$. Then, in complete analogy to the Standard Model, we postulate the absence of unbounded from below directions in the potential. Furthermore, and also in analogy to the Standard Model, we require that one mass squared, say $m_{11}^{2}$, is negative, to allow the spontaneous breaking of the electroweak symmetry. Lastly, a necessary condition for the decoupling of the second Higgs is $m_{22}^{2} \gg\left|m_{11}^{2}\right| / \lambda_{1}>0$ [12].

To study the minima of the potential, we will exploit the $S U(2)_{L}$ invariance to express the Higgs fields as

$$
\Phi_{1}=\frac{1}{\sqrt{2}}\left(\begin{array}{c}
0 \\
\varphi_{1}
\end{array}\right) \quad \Phi_{2}=\frac{1}{\sqrt{2}}\left(\begin{array}{c}
\sigma \\
\varphi_{2}
\end{array}\right),
$$

where $\sigma$ and $\varphi_{2}$ are complex fields, while $\varphi_{1}$ is a real field.

We consider now the direction in field space $\varphi_{2}=a \varphi_{1}, \sigma=b \varphi_{1}$, along which the potential reads:

$$
V_{a, b}\left(\varphi_{1}\right)=\frac{1}{2} m_{11}^{2}\left|\varphi_{1}\right|^{2}+\frac{1}{2} m_{22}^{2}\left(|a|^{2}+|b|^{2}\right)\left|\varphi_{1}\right|^{2}+\text { const. } \times\left|\varphi_{1}\right|^{4}
$$

Clearly, for any minimum of $V$ with vacuum expectation values $\left\langle\varphi_{1}\right\rangle,\left\langle\varphi_{2}\right\rangle,\langle\sigma\rangle$ there is a choice of the parameters $a$ and $b$ such that $V_{a, b}\left(\varphi_{1}\right)$ has a minimum at $\left\langle\varphi_{1}\right\rangle$, concretely for $a=\left\langle\varphi_{2}\right\rangle /\left\langle\varphi_{1}\right\rangle$ and $b=\langle\sigma\rangle /\left\langle\varphi_{1}\right\rangle$. This is only possible, though, if the quadratic part of eq. (3) is negative, thus for any minimum of $V$ it must hold that:

$$
\left|\left\langle\varphi_{2}\right\rangle\right|^{2}+|\langle\sigma\rangle|^{2}<\frac{\left|\left\langle\varphi_{1}\right\rangle\right|^{2}}{m_{22}^{2}}\left|m_{11}^{2}\right| .
$$

Utilizing this inequality it is straightforward to determine the minimum of $V$. The differentiation with respect to $\varphi_{1}$ yields

$$
\left|\left\langle\varphi_{1}\right\rangle\right|^{2}=\frac{2\left|m_{11}^{2}\right|}{\lambda_{1}}\left[1+\mathcal{O}\left(\sqrt{\frac{\left|m_{11}^{2}\right|}{\lambda_{1} m_{22}^{2}}}\right)\right] .
$$

Varying now $V$ with respect to $\sigma$ and $\varphi_{2}$, it can be checked that

$$
\begin{aligned}
& \langle\sigma\rangle=0, \\
& \left\langle\varphi_{2}\right\rangle \simeq-\left\langle\varphi_{1}\right\rangle \frac{\lambda_{6}^{*}\left\langle\varphi_{1}\right\rangle^{2}}{2 m_{22}^{2}},
\end{aligned}
$$

which shows that the electric charge is conserved. Furthermore, as the decoupling limit is approached, the Standard Model vacuum is recovered. 
Apart from recovering the nice features of the Standard Model vacuum, when taking the decoupling limit also the successful predictions of the Standard Model in electroweak observables and flavour physics are recovered. Namely, the existence of an additional Higgs doublet introduces contributions to the oblique parameters $S, T$ and $U$ which can be in tension with the electroweak precision measurements. However, it can be shown that in the decoupling limit $S, T$ and $U$ scale with $\left|m_{11}^{2}\right| /\left(\lambda_{1} m_{22}^{2}\right)$ [13], thus bringing the oblique parameters within their experimentally allowed values for sufficiently heavy extra scalar states.

Besides, the general 2HDM induces in general too large rates for the flavour changing neutral currents and the lepton flavour violating processes. Whereas this problem can be alleviated by assuming concrete flavour structures of the Yukawa couplings [14, a simpler way to suppress altogether all new contributions to the flavour violating processes consists on assuming that the new scalar particles are all very heavy.

The general flavour dependent part of the Lagrangian reads

$$
-\mathcal{L}^{\text {Yuk }}=\left(Y_{e}^{a}\right)_{i j} \bar{l}_{L i} e_{R j} \Phi_{a}+\left(Y_{u}^{a}\right)_{i j} \bar{q}_{L i} u_{R j} \tilde{\Phi}_{a}+\left(Y_{d}^{a}\right)_{i j} \bar{q}_{L i} d_{R j} \Phi_{a}+\text { h.c. }
$$

where $i, j=1,2,3$ are flavour indices, $a=1,2$ is a Higgs index and $\tilde{\Phi}_{a}=i \tau_{2} \Phi_{a}^{*}$. It will be convenient in what follows to work in the Higgs basis where one of the Higgs fields, say $\Phi_{2}$, does not acquire a vacuum expectation value. In this basis, then, the Yukawa matrices $Y_{e, u, d}^{1}$ are proportional to the fermion mass matrices.

Consider for illustration the contribution of the second Higgs doublet to the process $\mu \rightarrow e \gamma$, whose rate is strongly constrained by experiments $\mathrm{BR}(\mu \rightarrow e \gamma)<1.2 \times$ $10^{-11}$ [15]. For a wide range of parameters, this process is dominated by the two loop Barr-Zee diagrams [16]. The leading contribution comes from the top quark unless $Y_{u 33}^{2}$ is small [17]. In the decoupling limit the branching ratio reads:

$$
\mathrm{BR}(\mu \rightarrow e \gamma) \simeq \frac{8 \alpha^{3}}{3 \pi^{3}} \frac{\left|Y_{e 12}^{2}\right|^{2}}{\left|Y_{e 22}^{1}\right|^{2}}\left|f\left(\frac{m_{t}^{2}}{m_{h}^{2}}\right) \cos \alpha-\frac{Y_{u 33}^{2}}{Y_{u 33}^{1}} \frac{m_{t}^{2}}{m_{H}^{2}} \log ^{2} \frac{m_{t}^{2}}{m_{H}^{2}}\right|^{2}
$$

Here, $m_{t}$ denotes the top quark mass, $f(z)$ is defined in [17] and evaluates $f(2) \approx 1$, and $\alpha$ is the Higgs mixing angle, which reads $\cos \alpha \simeq\left|\lambda_{6}\right| v^{2} / m_{H}^{2}$ in the decoupling limit. Thus, the stringent experimental bound on $\operatorname{BR}(\mu \rightarrow e \gamma)$ can be evaded, for the concrete flavour structure $Y_{e 12}^{2}=\sqrt{Y_{e 11}^{1} Y_{e 22}^{1}}$ and $Y_{u 33}^{2}=Y_{u 33}^{1}$, if the scale of the heavy Higgs $m_{H} \gtrsim 2 \mathrm{TeV}$. Clearly, the rate of $\mu \rightarrow e \gamma$ can always be suppressed, regardless of the flavour structure of the Yukawa couplings, by increasing sufficiently the mass of the extra scalar degrees of freedom.

In the quark sector, strong constraints come from the measurement of the mass difference in meson anti-meson systems, such as $B_{s}^{0}-\bar{B}_{s}^{0}$, which arise at tree level in the general 2HDM. In the decoupling limit, the $B_{s}^{0}-\bar{B}_{s}^{0}$ mass difference approximately reads:

$$
\Delta m_{B_{s}} \simeq\left|\Delta m_{B_{s}}^{\mathrm{SM}}+\frac{4}{3} m_{B_{s}} f_{B_{s}}^{2} P_{2}^{L R} \frac{Y_{d 23}^{2 *} Y_{d 32}^{2}}{m_{H}^{2}}\right|
$$


Where $f_{B}$ is the $B$-meson decay constant, $f_{B_{s}}=238.8 \pm 9.5 \mathrm{MeV}$ [18], $m_{B_{s}}$ is the $B_{s}$ meson mass, $m_{B_{s}}=5.37 \mathrm{GeV}$ [19], and the coefficient $P_{2}^{L R}$ includes the renormalization group evolution from the scale $M_{Z}$ to $\sim m_{B_{S}}$ and the hadronization of the quarks to mesons [20] and reads $P_{2}^{L R} \simeq 3.0$. Assuming $\left|Y_{d 23}^{2 *}\right| \approx\left|Y_{d 32}^{2}\right| \approx \sqrt{Y_{d 22}^{1} Y_{d 33}^{1}}$, we estimate that the 2HDM contribution to the $B_{s}^{0}-\bar{B}_{s}^{0}$ mass difference lies within the theoretical error of the SM calculation, $\Delta m_{B_{s}}^{\mathrm{SM}}=(135 \pm 20) \times 10^{-13} \mathrm{GeV}$ [21], for $m_{H} \gtrsim 3 \mathrm{TeV}$. As before, the $2 \mathrm{HDM}$ contribution to the meson-antimeson mixing can always be suppressed for a sufficiently large $m_{H}$, regardless of the flavour structure of the quark Yukawa couplings.

\section{Neutrino masses in a $2 \mathrm{HDM}$ extended with right- handed neutrinos}

We will consider in this paper an extension of the Standard Model consisting in adding one extra Higgs doublet and at least one right-handed neutrino, singlet under the Standard Model gauge group. We will not impose any discrete symmetry on the model. Then, compatible with this matter content, the most general Lagrangian reads:

$$
\mathcal{L}=\mathcal{L}^{\text {kin }}+\mathcal{L}^{\text {Yuk }}+\mathcal{L}^{\nu}-V
$$

where $\mathcal{L}^{\text {kin }}$ contains the kinetic terms, $\mathcal{L}^{\text {Yuk }}$ is the Yukawa Lagrangian for the Standard Model fermions, given in eq. (7), $V$ is the Higgs potential, given in eq. (11) and $\mathcal{L}^{\nu}$ is the part of the Lagrangian involving right-handed neutrinos, given by:

$$
-\mathcal{L}^{\nu}=\left(Y_{\nu}^{a}\right)_{i j} \bar{l}_{L i} \nu_{R j} \tilde{\Phi}_{a}-\frac{1}{2} M_{\mathrm{M} i j} \bar{\nu}_{R i}^{C} \nu_{R j}+\text { h.c. }
$$

This term contains a Yukawa coupling, which leads to Dirac neutrino masses, and a Majorana mass for the right-handed neutrinos, with a size which is a priori unrelated to the electroweak symmetry breaking scale.

We will assume that the mass scale of the right-handed neutrinos is much larger than the electroweak symmetry breaking scale and the mass of all the extra Higgs mass eigenstates $H^{0}, A^{0}, H^{ \pm}$, which we denote collectively by $m_{H}$. Hence, the right-handed neutrinos are decoupled, leading to the following effective operators:

$$
-\mathcal{L}^{\nu, \text { eff }}=+\frac{1}{2} \kappa_{i j}^{a b}\left(\bar{l}_{L i} \tilde{\Phi}_{a}\right)\left(\tilde{\Phi}_{b}^{T} l_{L j}^{C}\right)+\text { h.c. }
$$

where, at the scale of the lightest right-handed neutrino,

$$
\kappa^{a b}\left(M_{1}\right)=\left(Y_{\nu}^{a} M_{\mathrm{M}}^{-1} Y_{\nu}^{b T}\right)\left(M_{1}\right)
$$

Since we have chosen to work in the basis where $\left\langle\Phi_{1}^{0}\right\rangle=v / \sqrt{2},\left\langle\Phi_{2}^{0}\right\rangle=0$, the neutrino mass matrix at the scale of the lightest right-handed neutrino depends just on the coupling $\kappa^{11}$ :

$$
\mathcal{M}_{\nu}\left(M_{1}\right)=\frac{v^{2}}{2} \kappa^{11}\left(M_{1}\right)
$$


which is diagonalized in the standard way:

$$
\mathcal{M}_{\nu}=U^{*} \operatorname{diag}\left(m_{1}, m_{2}, m_{3}\right) U^{\dagger}
$$

These are not, however, the neutrino parameters measured by experiments, where the energies involved are much smaller than the right-handed Majorana mass scale. In order to compare the predictions of the model with low energy experiments we will make use of the Renormalization Group Equations (RGE) given in the Appendix, to run the effective couplings $\kappa^{a b}$ from the scale $M_{1}$ to the scale $m_{H}$. Below the scale $m_{H}$ the neutrino mass matrix runs with the RGEs of the Standard Model extended with massive Majorana neutrinos. Since in this framework the neutrino masses are hierarchical, the running will not introduce any new qualitative feature but will only modify the values of the mass eigenvalues and the entries of the leptonic mixing matrix by a small factor, proportional to the tau Yukawa coupling squared and to the small $\operatorname{logarithm} \log \left(m_{H} / M_{Z}\right)$ [22].

To emphasize the main features of the quantum corrections to the neutrino mass matrix, we will concentrate in what follows on a see-saw model with just one righthanded neutrino with mass $M_{\text {maj }}$. Then, with this assumption, the neutrino Yukawa couplings $Y_{\nu}^{a}$ are 3 -vectors. In this scenario the neutrino mass matrix at the scale of the right-handed neutrino mass $M_{\text {maj }}$ is given by:

$$
\left[\kappa^{11}\right]^{\text {tree }}=\frac{Y_{\nu}^{1} Y_{\nu}^{1 T}}{M_{\text {maj }}},
$$

which has rank 1 and thus only one non-vanishing eigenvalue.

On the other hand, quantum effects introduce corrections to the neutrino mass matrix yielding at low energies $\kappa^{11}\left(m_{H}\right)=\left[\kappa^{11}\right]^{\text {tree }}+\delta \kappa^{11}$, where the correction reads, in the leading-log approximation,

$$
\delta \kappa^{11}=-\frac{1}{16 \pi^{2}} \beta_{\kappa^{11}}\left(M_{\mathrm{maj}}\right) \log \frac{M_{\mathrm{maj}}}{m_{H}} .
$$

Using the explicit form of the $\beta$ function in the Appendix, it follows that this correction can be schematically written as:

$$
\delta \kappa^{11} \simeq B_{1 a} \kappa^{a 1}+\kappa^{1 a} B_{1 a}^{T}+b \kappa^{22}
$$

Here $B_{1 a}$ denote $3 \times 3$ matrices whereas $b$ is a number. The first two terms generalize the well known correction to the neutrino mass matrix in the Standard Model including the dimension-5 Weinberg operator. However, the term proportional to $\kappa^{22}$ does not have any correspondence in the Standard Model and, as we will see, introduces new qualitative features. The coefficient $b$ explicitly reads:

$$
b=-\frac{1}{16 \pi^{2}} 2 \lambda_{5} \log \frac{M_{\mathrm{maj}}}{m_{H}},
$$


which depends linearly on the coefficient of the potential term $\lambda_{5}\left(\Phi_{1}^{\dagger} \Phi_{2}\right)\left(\Phi_{1}^{\dagger} \Phi_{2}\right)$, while only logarithmically on the ratio between the scale of the right-handed neutrino and the overall scale of the extra scalars $H^{0}, H^{ \pm}, A^{0}$.

The neutrino mass matrix $\mathcal{M}_{\nu}=\left[\mathcal{M}_{\nu}\right]^{\text {tree }}+\delta \mathcal{M}_{\nu}$ can be diagonalized using perturbation theory, giving as a result the eigenvalues $m_{i}=m_{i}^{(0)}+\delta m_{i}$ with $m_{i}^{(0)}$ the eigenvalue at tree level and $\delta m_{i}$ the first order correction.

At lowest order in perturbation theory, taking into account only the tree level mass term, there is only one non-vanishing neutrino mass eigenvalue:

$$
m_{3}^{(0)}=\frac{v^{2}}{2 M_{\text {maj }}}\left|Y_{\nu}^{1}\right|^{2} .
$$

On the other hand, the third column of the leptonic mixing matrix reads;

$$
U_{i 3}^{(0)}=\frac{Y_{\nu i}^{1 *}}{\left|Y_{\nu}^{1}\right|}
$$

while the first two columns are undefined, due to the degeneracy of the corresponding neutrino mass eigenvalues. In this expression, $\left|Y_{\nu}^{a}\right|=\left(\sum_{i}\left|Y_{\nu i}^{a}\right|^{2}\right)^{1 / 2}$.

The correction to the neutrino mass eigenvalues due to the perturbation $\delta \kappa^{11}$ is given by:

$$
\delta m_{i}=\frac{v^{2}}{2} \operatorname{Re}\left[\left(U^{(0) T} \delta \kappa^{11} U^{(0)}\right)_{i i}\right]
$$

which slightly modifies the value of the heaviest neutrino mass eigenvalue:

$$
\delta m_{3}=\frac{v^{2}}{2 M_{\text {maj }}} \operatorname{Re}\left[2\left(Y_{\nu}^{1 \dagger} B_{1 a} Y_{\nu}^{a}\right)+b \frac{\left(Y_{\nu}^{1 \dagger} Y_{\nu}^{2}\right)^{2}}{\left|Y_{\nu}^{1}\right|^{2}}\right] .
$$

More importantly, this correction is also non-vanishing for $\delta m_{2}$, thus generating radiatively a second neutrino mass eigenvalue. This is in contrast to the widely studied case of the Standard Model extended with a single right-handed neutrino, where there is only one non-vanishing neutrino mass eigenvalue, even after taking into account the renormalization group running. 1

To show this, we write explicitly the radiative correction to the next-to-lightest neutrino mass eigenvalue:

$$
\delta m_{2}=\frac{v^{2}}{2} \operatorname{Re}\left[U_{p 2}^{(0)} \delta \kappa_{p q}^{11} U_{q 2}^{(0)}\right]
$$

which crucially depends on the second column of the zero-th order leptonic mixing matrix. Since the matrix $U^{(0)}$ is unitary, the vector $U_{q 2}^{(0)}$ should satisfy $\sum_{q} U_{q 2}^{(0)} U_{q 3}^{(0) *}=0$, $\sum_{q} U_{q 2}^{(0)} U_{q 2}^{(0) *}=1$. A vector that satisfies those properties can be easily constructed from

\footnotetext{
${ }^{1}$ There are, however, tiny finite corrections arising from two-loop diagrams involving $\mathrm{W}$ bosons 23 .
} 
the vectors $Y_{\nu}^{1}$ and $Y_{\nu}^{2}$ using the Gram-Schmidt process. Starting with $U_{i 3}^{(0)}$ given by eq. (21) one finds:

$$
U_{i 2}^{(0)}=\frac{1}{N_{2}}\left[Y_{\nu i}^{2 *}-\frac{Y_{\nu}^{2 \dagger} Y_{\nu}^{1}}{\left|Y_{\nu}^{1}\right|} \frac{Y_{\nu i}^{1 *}}{\left|Y_{\nu}^{1}\right|}\right] e^{-\frac{i}{2} \arg \left(-\lambda_{5}\right)},
$$

where

$$
N_{2}=\left[Y_{\nu}^{2 \dagger} Y_{\nu}^{2}-\frac{\left|Y_{\nu}^{2 \dagger} Y_{\nu}^{1}\right|^{2}}{\left|Y_{\nu}^{1}\right|^{2}}\right]^{1 / 2}
$$

Substituting into the expression for $\delta m_{2}$ we find

$$
m_{2}=\frac{1}{16 \pi^{2}} \frac{\left|\lambda_{5}\right| v^{2}}{M_{\mathrm{maj}}}\left[\left|Y_{\nu}^{2}\right|^{2}-\frac{\left|Y_{\nu}^{2 \dagger} Y_{\nu}^{1}\right|^{2}}{\left|Y_{\nu}^{1}\right|^{2}}\right] \log \frac{M_{\mathrm{maj}}}{m_{H}} .
$$

(Note that the phase in eq. (25) has been chosen to yield $m_{2}$ real and positive.) It is apparent from this expression that in order to generate a non-vanishing neutrino mass eigenvalue it is necessary the misalignment between the Yukawa couplings $Y_{\nu}^{1}$ and $Y_{\nu}^{2}$, or in more physical terms, it is necessary the existence of new sources of flavour violation in the neutrino sector. These new sources necessarily generate, through quantum corrections, off-diagonal elements in the charged lepton Yukawa coupling $Y_{e}^{2}$, which in turn induce a contribution to the lepton flavour violating processes. Nevertheless, as explained in Section 2 this contribution is suppressed by the large mass of the extra Higgs particles, and can be consistent with experiments if the extra particles are sufficiently heavy.

Furthermore, it is interesting to note that, under some well motivated assumptions, the hierarchy between the tree level mass $m_{3}$ and the radiatively generated neutrino mass $m_{2}$ can be fairly mild. For instance, taking the typical values $\left|\lambda_{5}\right| \sim 1, M_{\text {maj }} \sim 10^{11} \mathrm{GeV}$ and $m_{H} \sim 1 \mathrm{TeV}$ and assuming non-aligned neutrino Yukawa couplings with $\left|Y_{\nu}^{2}\right| \sim\left|Y_{\nu}^{1}\right|$ one obtains for the ratio between the two heaviest neutrino mass eigenvalues:

$$
\frac{m_{2}}{m_{3}} \simeq \frac{\left|\lambda_{5}\right|}{8 \pi^{2}} \frac{\left|Y_{\nu}^{2}\right|^{2}}{\left|Y_{\nu}^{1}\right|^{2}} \log \frac{M_{\mathrm{maj}}}{m_{H}} \sim 0.2,
$$

which yields a mild mass hierarchy, in qualitative agreement with the experimental data. Note that, whereas the overall scale of the light neutrino masses depends linearly on the inverse of the heavy right-handed neutrino mass, the ratio between the two heaviest neutrino mass eigenvalues depends only logarithmically with the masses of the new particles. As a consequence, the result in eq. (28) is fairly insensitive to the exact values of the masses of the heavy particles.

In the previous analysis we have assumed for simplicity that only one right-handed neutrino participates in the neutrino mass generation. In the more realistic case where there are several right-handed neutrinos, the tree level contributions to all neutrino mass eigenvalues will be non-vanishing. Nevertheless, as discussed in the Introduction, if the 
neutrino Yukawa coupling $Y_{\nu}^{1}$ has hierarchical eigenvalues, as suggested by the observed hierarchies in the quark and charged lepton masses, then the neutrino mass hierarchy generated (at tree level) by the see-saw mechanism is in general several orders of magnitude larger than the one inferred from experiments. Therefore, the radiatively generated contribution to the next-to-lightest neutrino masses by the presence of the second Higgs doublet will dominate over the tree level contribution, and the conclusions presented above will still hold.

In an extended scenario with $N_{H}$ Higgs doublets and one right-handed neutrino, the radiatively induced next-to-lightest neutrino mass receives $N_{H} \times\left(N_{H}-1\right) / 2$ contributions, each of them proportional to the coefficient of the term $\lambda_{5}^{a b}\left(\Phi_{a}^{\dagger} \Phi_{1}\right)\left(\Phi_{b}^{\dagger} \Phi_{1}\right), a, b=2 \ldots N_{H}$, in the Higgs potential. Therefore, in this case the radiatively generated neutrino mass is enhanced.

It is amusing to speculate that adding more Higgs doublets to the particle content of the model may also be relevant to understand the observed pattern of neutrino mixing angles. In a model with $N_{H}$ Higgs doublets, only the Higgs that acquires a vacuum expectation value, $\Phi_{1}$, will contribute to the tree level mass. Assuming that this is

the largest mass, $m_{3}=\sqrt{\Delta m_{\text {atm }}^{2}}$, it follows from eq. (21) that $U_{i 3} \propto Y_{\nu i}^{1 *}$. Therefore, if there is any pattern in the neutrino Yukawa coupling $Y_{\nu i}^{1}$, stemming e.g. from an underlying flavour symmetry, then this pattern will be inherited by $U_{i 3}$, thus providing an explanation to the apparent structure of the last column of the leptonic mixing angle: $\left|U_{13}\right| \simeq 0,\left|U_{23}\right| \simeq\left|U_{33}\right|$. In contrast, there are $N_{H}-1$ Higgses which contribute via quantum effects to the generation of the solar neutrino mass scale, $m_{2}=\sqrt{\Delta m_{\text {sol }}^{2}}$ and of the second column of the leptonic mixing matrix, $U_{i 2}$. As a consequence, even if there is a structure in each of the neutrino Yukawa couplings, $Y_{\nu i}^{a}, a=2, \ldots N_{H}$, the generated $U_{i 2}$ will be structureless, since it receives contributions from all these Yukawa couplings. This is in rough agreement with observations, which reveal that the three entries in $U_{i 2}$ are all $\mathcal{O}(0.1)$, without displaying any remarkable structure (or, alternatively, the solar angle is neither maximal nor zero). Therefore, in the Standard Model extended with righthanded neutrinos and several Higgs doublets, the last column of the leptonic mixing matrix is expected to display a "hierarchical" structure, whereas the second column, an "anarchical" structure 24], in qualitative agreement with the data.

\section{Comparison to the two right-handed neutrino model}

The scenario discussed in this paper leads to a dimension-5 operator which is identical to the one generated by the Standard Model (with a single Higgs doublet) extended by two heavy right-handed neutrinos. There are however some conceptual differences in the way these two scenarios reproduce the observed neutrino data, which we discuss here.

Let us first demonstrate the equivalence of the two Higgs doublet model extended with one right-handed neutrino (2HD-1RHN model) and the Standard model extended 
with two right-handed neutrinos (1HD-2RHN model). At low energies, the coefficient of the dimension five operator generated in the $2 \mathrm{HD}-1 \mathrm{RHN}$ model reads, following eqs. (13), (18),

$$
\kappa^{11}\left(m_{H}\right) \simeq \frac{1}{M_{\text {maj }}}\left[Y_{\nu}^{1} Y_{\nu}^{1^{T}}+B_{1 a} Y_{\nu}^{a} Y_{\nu}^{1^{T}}+Y_{\nu}^{1} Y_{\nu}^{a T} B_{1 a}^{T}+b Y_{\nu}^{2} Y_{\nu}^{2^{T}}\right]
$$

which can be recast as

$$
\kappa^{11}\left(m_{H}\right) \simeq \frac{1}{M_{\mathrm{maj}}}\left[\left(Y_{\nu}^{1}+B_{1 a} Y_{\nu}^{a}\right)\left(Y_{\nu}^{1}+B_{1 a} Y_{\nu}^{a}\right)^{T}-B_{1 a} Y_{\nu}^{a} Y_{\nu}^{a^{T}} B_{1 a^{\prime}}^{T}+b Y_{\nu}^{2} Y_{\nu}^{2 T}\right]
$$

Neglecting the term of $\mathcal{O}\left(B^{2}\right)$ and defining $\tilde{Y}_{\nu}^{1}=Y_{\nu}^{1}+B_{1 a} Y_{\nu}^{a}$, one obtains the following low energy neutrino mass matrix

$$
\mathcal{M}_{\nu} \simeq\left(\frac{\tilde{Y}_{\nu}^{1} \tilde{Y}_{\nu}^{1 T}}{M_{\text {maj }}}+\frac{b Y_{\nu}^{2} Y_{\nu}^{2 T}}{M_{\text {maj }}}\right) \frac{v^{2}}{2}
$$

This result is formally identical to the effective operator which arises in the low energy limit of the 1HD-2RHN model. More specifically, in this model the high energy Lagrangian reads, in the basis where the $2 \times 2$ right-handed neutrino mass matrix is diagonal,

$$
-\mathcal{L}^{\nu, 2 \mathrm{RHN}}=\left(Y_{\nu}\right)_{i j} \bar{l}_{L i} \nu_{R j} \tilde{\Phi}-\frac{1}{2} M_{1} \bar{\nu}_{R 1}^{C} \nu_{R 1}-\frac{1}{2} M_{2} \bar{\nu}_{R 2}^{C} \nu_{R 2}+\text { h.c. }
$$

which leads, when $M_{1}, M_{2} \gg v$, to the effective neutrino mass matrix

$$
\mathcal{M}_{\nu}^{2 \mathrm{RHN}} \simeq\left(\frac{Y_{1} Y_{1}^{T}}{M_{1}}+\frac{Y_{2} Y_{2}^{T}}{M_{2}}\right) \frac{v^{2}}{2}
$$

being $Y_{1,2}$ column vectors defined as $Y_{1} \equiv\left(Y_{\nu}\right)_{i 1}, Y_{2} \equiv\left(Y_{\nu}\right)_{i 2}$. By comparing eqs. (31) and (33) it follows that, from the point of view of the neutrino mass generation, the 2HD$1 \mathrm{RHN}$ model is equivalent to the $1 \mathrm{HD}-2 \mathrm{RHN}$ model with the following correspondence among parameters

$$
\left\{Y_{1}, Y_{2}, M_{1}, M_{2}\right\} \leftrightarrow\left\{\tilde{Y}_{\nu}^{1}, Y_{\nu}^{2}, M_{\text {maj }}, M_{\text {maj }} / b\right\}
$$

This correspondence allows to write explicit expressions for the most general Yukawa couplings $Y_{\nu}^{1}, Y_{\nu}^{2}$ which lead to the neutrino masses $m_{2}, m_{3}$ and the leptonic mixing matrix $U$. Using the results of [25], one easily finds:

$$
\begin{aligned}
& \tilde{Y}_{\nu}^{1}=\frac{\sqrt{2}}{v} \sqrt{M_{\mathrm{maj}}}\left(\sqrt{m_{2}} \cos \hat{\theta} U_{i 2}^{*} \pm \sqrt{m_{3}} \sin \hat{\theta} U_{i 3}^{*}\right), \\
& Y_{\nu}^{2}=\frac{\sqrt{2}}{v} \sqrt{\frac{M_{\mathrm{maj}}}{b}}\left(-\sqrt{m_{2}} \sin \hat{\theta} U_{i 2}^{*} \pm \sqrt{m_{3}} \cos \hat{\theta} U_{i 3}^{*}\right),
\end{aligned}
$$


where $\hat{\theta}$ is a complex angle which parametrizes the family of Yukawa couplings compatible with the low energy neutrino data (note that this parametrization may fail if the term of $\mathcal{O}\left(B^{2}\right)$ in eq. (30) can not be neglected). Finally, the Yukawa coupling with the Higgs $\Phi_{1}$ is $Y_{\nu}^{1}=\left(1-B_{11}\right) \tilde{Y}_{\nu}^{1}-B_{12} Y_{\nu}^{2}$.

Furthermore, this correspondence allows to better appreciate the advantages of the 2HD-1RHN model over the 1HD-2RHN model in the generation of a mild mass hierarchy. In the 1HD-2RHN model, the neutrino mass hierarchy is essentially given by

$$
\frac{m_{3}}{m_{2}} \sim \frac{\left|Y_{2}\right|^{2}}{\left|Y_{1}\right|^{2}} \frac{M_{1}}{M_{2}}
$$

therefore the only possibilities to generate a mild neutrino mass hierarchy are i) $\left|Y_{2}\right|^{2} \sim$ $\left|Y_{1}\right|^{2}, M_{2} \sim M_{1}$, or ii) $\left|Y_{2}\right| \gg\left|Y_{1}\right|$ with $M_{2} / M_{1} \sim\left|Y_{1}\right|^{2} /\left|Y_{2}\right|^{2}$. In view of the observed large hierarchies in the quark and charged lepton Yukawa eigenvalues, in a model with two right-handed neutrinos one expects $\left|Y_{2}\right| \gg\left|Y_{1}\right|$, which hence requires a huge hierarchy between the two right-handed neutrino masses in order to render a mild light neutrino mass hierarchy. More concretely, if the neutrino Yukawa eigenvalues have a similar hierarchy as the up-type quark masses, $\left|Y_{2}\right| /\left|Y_{1}\right| \sim m_{t} / m_{c} \sim 150$, then it is required $M_{2} / M_{1} \sim 20000$. On the other hand, if the hierarchy is similar to the down-type quark masses, $\left|Y_{2}\right| /\left|Y_{1}\right| \sim m_{b} / m_{s} \sim 40$, then it is required $M_{2} / M_{1} \sim 1600$. Whereas such large hierarchies in the right-handed neutrino masses cannot be precluded, it is difficult to conceive that in the decoupling limit the large hierarchies in the Yukawa couplings cancel almost exactly a huge hierarchy in the right-handed neutrino masses to generate at low energies a light neutrino mass hierarchy of $\sim 6$, as inferred from experiments.

This drawback is very naturally circumvented by the $2 \mathrm{HD}-1 \mathrm{RHN}$ model which as we argued above is equivalent, concerning the neutrino mass generation, to the $1 \mathrm{HD}-2 \mathrm{RHN}$ model. In the equivalent 1HD-2RHN model, the Yukawa couplings $Y_{1}$ and $Y_{2}$ are naturally of the same size, since in the original $2 \mathrm{HD}-1 \mathrm{RHN}$ they correspond to Yukawa couplings to the same generation of right-handed neutrinos. Furthermore, in the corresponding two right-handed neutrino model, the masses $M_{1}$ and $M_{2}$ naturally present a mild hierarchy, given by the factor $1 / b \sim \mathcal{O}(1-10)$. As a result, the $2 \mathrm{HD}-1 \mathrm{RHN}$ is equivalent to a 1HD-2RHN model which naturally fulfills the conditions i) to reproduce the observed mild neutrino mass hierarchy.

Another important difference between the 1HD-2RHN model and the 2HD-1RHN model concerns the possibility of observing other phenomena at low energies apart from neutrino masses. It is well known that, in its simplest version, the $1 \mathrm{HD}-2 \mathrm{RHN}$ model does not have any other observable low energy prediction apart from the tininess of neutrino masses. In this model the scale of lepton flavour and lepton number violation both coincide with the scale of the right-handed Majorana neutrino masses, which are postulated to be much larger than the electroweak symmetry breaking scale. As a consequence, the rates of all flavour and lepton number violating processes are inversely proportional to the heavy right-handed neutrino mass resulting in tiny rates.2 In contrast, in the

\footnotetext{
${ }^{2} \mathrm{~A}$ notable exception is the $1 \mathrm{HD}-2 \mathrm{RHN}$ scenario where the right-handed neutrinos form a pseudo-
} 
2HD-1RHN model, apart from the lepton flavour associated to the right-handed neutrino couplings there exist another source of lepton flavour violation associated to the charged lepton couplings to the second Higgs doublet, inducing rates for the rare lepton decays suppressed by the heavy Higgs masses. If the additional scalar degrees of freedom have masses not far from the electroweak symmetry breaking scale, the induced rates of the lepton flavour violating processes could be large enough to be observed in experiments. A more detailed discussion about the prospects to observe the process $\mu \rightarrow e \gamma$ in experiments will be presented in Section 6 .

\section{Corrections to the mixing angles and discussion of $\sin \theta_{13}$}

Below the right-handed neutrino mass scale, the neutrino mixing angles receive radiative corrections with two different origins. First, the change in the entries of the neutrino mass matrix due to the RGE running, eq. (18), generates a correction to the leptonic mixing matrix given by:

$$
\delta U_{\kappa}=U^{(0)} T
$$

where

$$
\begin{aligned}
T_{i i} & \equiv-\frac{i\left[U^{(0) T} \delta \mathcal{M}_{\nu} U^{(0)}\right]_{i i}}{2 m_{i}^{(0)}} \\
T_{i j} & \equiv \frac{m_{i}^{(0)}\left[U^{(0) T} \delta \mathcal{M}_{\nu} U^{(0)}\right]_{i j}+m_{j}^{(0)}\left[U^{(0) T} \delta \mathcal{M}_{\nu} U^{(0)}\right]_{i j}^{*}}{m_{j}^{(0) 2}-m_{i}^{(0) 2}} \text { if } i \neq j .
\end{aligned}
$$

However, this is not the physical leptonic mixing matrix measured by experiments, since the RGE running also modifies the structure of the charged lepton Yukawa couplings. More specifically, if the charged lepton Yukawa coupling $Y_{e}^{1}$ is diagonal at $M_{\text {maj }}$, the radiative corrections induced by $Y_{e}^{2}$ will generate at low energies off-diagonal entries in $Y_{e}^{1}$. It is then necessary to redefine the charged lepton fields in order to render a diagonal charged lepton Yukawa coupling, namely $l_{L} \rightarrow V_{e}^{L} l_{L}, e_{R} \rightarrow V_{e}^{R} e_{R}$, where $V_{e}^{L}$, $V_{e}^{R}$ follow from the singular value decomposition, $Y_{e}^{1}=V_{e}^{L} \operatorname{diag}\left(y_{e 1}^{1}, y_{e 2}^{1}, y_{e 3}^{1}\right) V_{e}^{R \dagger}$. This redefinition introduces an additional correction to the leptonic mixing matrix given by

$$
\delta U_{Y_{e}}=\left(V_{e}^{L}-\mathbb{1}\right)^{T} U^{(0)} .
$$

Dirac pair with masses $\mathcal{O}(100-1000) \mathrm{GeV}$. In this case, the Yukawa couplings can be sizable while correctly reproducing the tininess of the neutrino masses. As a consequence, the rates for $\mu \rightarrow e \gamma$ and neutrinoless double beta decay can be largely enhanced, possibly allowing their observation in the next round of experiments $[26$. 
The matrix $V_{e}^{L}$ can be explicitly calculated from the $\beta$-functions of the charged lepton Yukawa couplings. Using

$$
\left.Y_{e}^{1} Y_{e}^{1 \dagger}\right|_{m_{H}}=Y_{e}^{1} Y_{e}^{1 \dagger}-\frac{1}{16 \pi^{2}}\left(\beta_{Y_{e}^{1}} Y_{e}^{1 \dagger}+Y_{e}^{1} \beta_{Y_{e}^{1}}^{\dagger}\right) \log \frac{M_{\mathrm{maj}}}{m_{H}},
$$

we obtain

$$
\left(V_{e}^{L}\right)_{i j}=-\frac{1}{16 \pi^{2}} \frac{\left(\beta_{Y_{e}^{1}} Y_{e}^{1 \dagger}+Y_{e}^{1} \beta_{Y_{e}^{1}}^{\dagger}\right)_{i j}}{\left(y_{e j}^{1}\right)^{2}-\left(y_{e i}^{1}\right)^{2}} \log \frac{M_{\mathrm{maj}}}{m_{H}} \quad i \neq j .
$$

Therefore, summing up the two contributions, the leptonic mixing matrix at low energies reads, in the physical basis,

$$
U^{(1)}=V_{e}^{L T} U^{(0)}+U^{(0)} T .
$$

We are particularly interested in the correction to the last column of the leptonic mixing matrix, which in general yields a non-vanishing contribution to $\sin \theta_{13}$ and a deviation from the maximal atmospheric mixing which may be observed in experiments. Concretely, the correction to the third column of the leptonic mixing matrix due to the running of $\kappa$ reads

$$
\begin{aligned}
\left(\delta U_{\kappa}\right)_{i 3}=\left(U^{(0)} T\right)_{i 3}= & \frac{Y_{\nu i}^{1 *}}{\left|Y_{\nu}^{1}\right|}\left[-\frac{\operatorname{Re}\left(Y_{\nu}^{1 \dagger} B_{1 a} Y_{\nu}^{a}\right)}{\left|Y_{\nu}^{1}\right|^{2}}+\frac{i}{2} \frac{\operatorname{Im}\left(b^{*}\left(Y_{\nu}^{2 \dagger} Y_{\nu}^{1}\right)^{2}\right)}{\left|Y_{\nu}^{1}\right|^{4}}\right] \\
& +\frac{\left(B_{1 a}^{*} Y_{\nu}^{a *}\right)_{i}}{\left|Y_{\nu}^{1}\right|}+\left(Y_{\nu i}^{2 *}-Y_{\nu i}^{1 *} \frac{\left(Y_{\nu}^{2 \dagger} Y_{\nu}^{1}\right)}{\left|Y_{\nu}^{1}\right|^{2}}\right) b^{*} \frac{Y_{\nu}^{2 \dagger} Y_{\nu}^{1}}{\left|Y_{\nu}^{1}\right|^{3}}
\end{aligned}
$$

while the contribution from the rediagonalization of the charged lepton Yukawa coupling reads:

$$
\left(\delta U_{Y_{e}}\right)_{i 3}=-\frac{1}{16 \pi^{2}} \sum_{j \neq i} \frac{\left(\beta_{Y_{e}^{1}} Y_{e}^{1 \dagger}+Y_{e}^{1} \beta_{Y_{e}^{1}}^{\dagger}\right)_{j i}}{\left(y_{e i}^{1}\right)^{2}-\left(y_{e j}^{1}\right)^{2}} \frac{Y_{\nu j}^{1 *}}{\left|Y_{\nu}^{1}\right|} \log \frac{M_{\text {maj }}}{m_{H}} .
$$

A quantity of particular interest is the angle $\theta_{13}$, which is constrained by present experiments to be small. It is interesting that radiative corrections can generate in this model a fairly large value of $\theta_{13}$, possibly at the reach of the planned experiments, even if its tree-level value vanishes. Summing up the contributions from eqs. (45) and (46), and neglecting terms cubic in the charged lepton Yukawa couplings, we obtain that the radiatively induced value of $U_{13}$ is

$$
\begin{aligned}
\delta U_{13}= & -\frac{1}{16 \pi^{2}} \frac{Y_{\nu 1}^{2 *}}{\left|Y_{\nu}^{1}\right|}\left[3 \operatorname{Tr}\left(Y_{u}^{1 \dagger} Y_{u}^{2}+Y_{d}^{1} Y_{d}^{2 \dagger}\right)+2 \lambda_{6}^{*}+2 \lambda_{5}^{*} \frac{Y_{\nu}^{2 \dagger} Y_{\nu}^{1}}{\left|Y_{\nu}^{1}\right|^{2}}\right] \log \frac{M_{\text {maj }}}{m_{H}} \\
& +\frac{1}{16 \pi^{2}} \frac{\left(Y_{\nu}^{1 \dagger}\left(Y_{e}^{1}\right)^{-1} Y_{e}^{2 \dagger}\right)_{1}}{\left|Y_{\nu}^{1}\right|}\left[3 \operatorname{Tr}\left(Y_{u}^{2 \dagger} Y_{u}^{1}+Y_{d}^{2} Y_{d}^{1 \dagger}\right)\right] \log \frac{M_{\text {maj }}}{m_{H}}
\end{aligned}
$$


which is, as the ratio $m_{2} / m_{3}$, suppressed by the loop factor but enhanced by the large logarithm of the ratio of the Majorana mass over the heavy Higgs mass. As a result, the radiatively generated $\theta_{13}$ can be as large as $\sim 0.2$ if any of the entries in the bracket is $\sim \mathcal{O}(1)$.

So far we have considered only the corrections to the neutrino mixing angles from the running below the right-handed neutrino mass scale. However, in a large class of models the cut-off of the theory lies at higher energies and additional contributions to the leptonic mixing may arise from radiative corrections between the cut-off scale $\Lambda$ and the right-handed neutrino mass scale $M_{\text {maj }}$. The corrections for this case can be derived using eqs. (45), (46) and the substitution rules given in Appendix A.2, the result being:

$$
\begin{aligned}
\delta U_{13}= & -\frac{Y_{\nu 1}^{2 *}}{\left|Y_{\nu}^{1}\right|}\left\{\left[\operatorname{Tr}\left(3 Y_{u}^{1 \dagger} Y_{u}^{2}+3 Y_{d}^{1} Y_{d}^{2 \dagger}+Y_{\nu}^{1 \dagger} Y_{\nu}^{2}\right)+2 Y_{\nu}^{1 \dagger}\left(Y_{e}^{1}\right)^{-1} Y_{e}^{2 \dagger} Y_{\nu}^{1}\right] \frac{\log \frac{\Lambda}{M_{\text {maj }}}}{16 \pi^{2}}\right. \\
& \left.+\left[3 \operatorname{Tr}\left(Y_{u}^{1 \dagger} Y_{u}^{2}+Y_{d}^{1} Y_{d}^{2 \dagger}\right)+2 \lambda_{6}^{*}+2 \lambda_{5}^{*} \frac{Y_{\nu}^{2 \dagger} Y_{\nu}^{1}}{\left|Y_{\nu}^{1}\right|^{2}}\right] \frac{\log \frac{M_{\text {maj }}}{m_{H}}}{16 \pi^{2}}\right\} \\
+ & \frac{\left(Y_{\nu}^{1 \dagger}\left(Y_{e}^{1}\right)^{-1} Y_{e}^{2 \dagger}\right)}{\left|Y_{\nu}^{1}\right|}\left\{\operatorname{Tr}\left(Y_{\nu}^{2 \dagger} Y_{\nu}^{1}\right) \frac{\log \frac{\Lambda}{M_{\text {maj }}}}{16 \pi^{2}}+3 \operatorname{Tr}\left(Y_{u}^{2 \dagger} Y_{u}^{1}+Y_{d}^{2} Y_{d}^{1 \dagger}\right) \frac{\log \frac{\Lambda}{m_{H}}}{16 \pi^{2}}\right\} .
\end{aligned}
$$

Quantum effects also induce corrections to the atmospheric mixing angle, leading to deviations to the maximal mixing even if $\theta_{23}=\pi / 4$ at tree level. It is interesting that if the neutrino Yukawa couplings are the dominant source of flavour violation in the leptonic sector, then a correlation arises between the deviations of $U_{23} / U_{33}$ and $U_{13}$ from their corresponding values at the cut-off scale.

In this limit, the radiative corrections to the last column of the leptonic mixing matrix are dominated by the first line of eq. (47), which can be schematically written as:

$$
U_{i 3}=\left(1+\epsilon_{3}\right) U_{i 3}^{(0)}+\epsilon_{2} U_{i 2}^{(0)} .
$$

It can be checked that to first order the ratio $U_{23} / U_{33}$ does not depend on $\epsilon_{3}$. Then, using the equation for $U_{13}$ to eliminate $\epsilon_{2}$ it follows that:

$$
\frac{U_{23}}{U_{33}}-\frac{U_{23}^{(0)}}{U_{33}^{(0)}} \simeq \frac{U_{22}^{(0)} U_{33}^{(0)}-U_{32}^{(0)} U_{23}^{(0)}}{U_{33}^{(0) 2}} \frac{U_{13}-U_{13}^{(0)}}{U_{12}^{(0)}} .
$$

Concretely, in the case when at the cut-off scale the atmospheric mixing angle is exactly maximal and $\theta_{13}$ vanishes, at low energies the elements of the leptonic mixing matrix approximately satisfy

$$
\frac{U_{23}}{U_{33}}-1 \simeq 2 \sqrt{2} U_{13},
$$

which can be recast as

$$
\tan \theta_{23} \simeq\left|1+2 \sqrt{2} \sin \theta_{13} e^{-i \delta}\right| \quad \text { or } \quad \theta_{23}-\frac{\pi}{4} \simeq \sqrt{2} \sin \theta_{13} \cos \delta .
$$



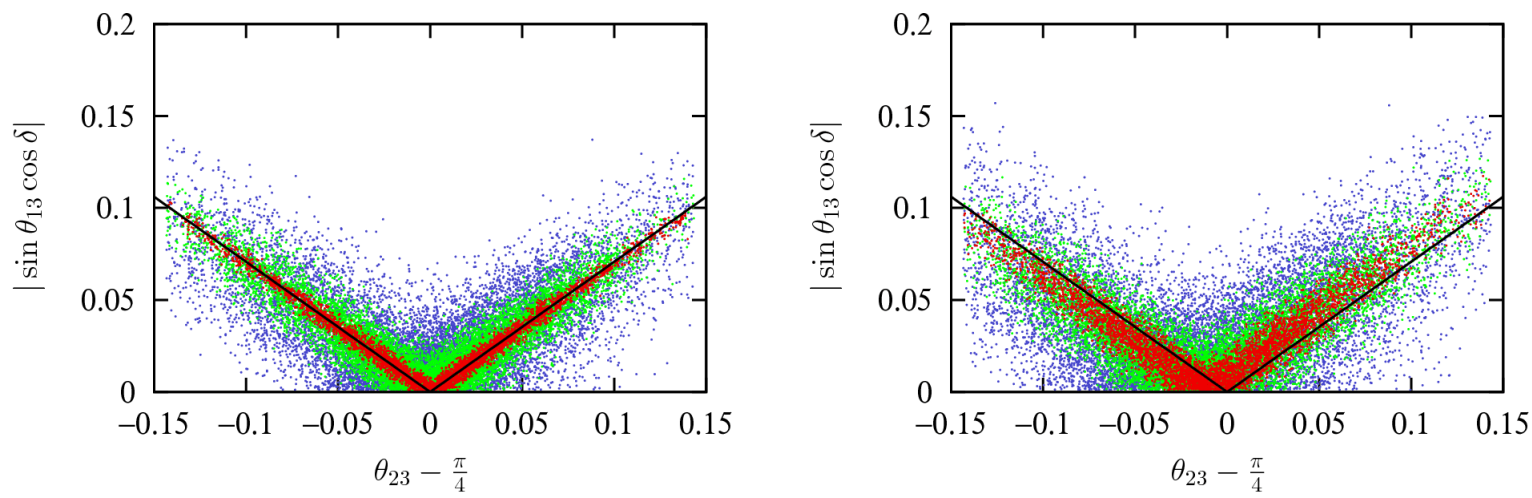

Figure 1: Scatter plots showing $\left|\sin \theta_{13} \cos \delta\right|$ against $\theta_{23}-\frac{\pi}{4}$ at low energies for random choices of high energy parameters consistent with the measured neutrino oscillation parameters. We have assumed tri-bi-maximal mixing at the cut-off scale, being the deviation from $\theta_{23}=\pi / 4$ and $\theta_{13}=0$ at low energies only due to the radiative corrections as described in the main text. The cut-off scale is $\Lambda=M_{\text {maj }}=10^{14} \mathrm{GeV}$ in the left panel and $\Lambda=10^{18} \mathrm{GeV}$ in the right panel.

If there are additional sources of lepton flavour violation, then the low energy values of $\theta_{23}$ and $\theta_{13}$ are expected to deviate from this relation. This is illustrated in the scatter plots shown in fig. 1, which have been obtained by the numerical one loop integration of the RGEs of the $2 \mathrm{HDM}$ extended by one right-handed neutrino. We assume in the plot $m_{H}=3 \mathrm{TeV}, M_{\text {maj }}=10^{14} \mathrm{GeV}$ and tri-bi-maximal mixing at a cut-off scale, which we take $\Lambda=M_{\text {maj }}\left(\Lambda=10^{18} \mathrm{GeV}\right)$ in the left (right) panel. Quantum effects generate a non-vanishing value for $\theta_{13}$ and $\theta_{23}-\frac{\pi}{4}$, mostly due to the RGE effects of $\lambda_{5}$ and $\lambda_{6}$, as follows from eqs. (47) and (48). In the plot we fix $\left|\lambda_{5}\right|=0.5$ and we take random values with $\left|\lambda_{6}\right|<0.45$, in order to preserve the perturbativity of the quartic couplings in the renormalization group running. To investigate the impact of the charged lepton mixing in the correlation we have adopted the ansatz $Y_{e}^{2}=V Y_{e}^{1}$, where $V$ is a general unitary matrix with random angles and phases. Furthermore, since the effect of the charged lepton Yukawa couplings on the corrections to the leptonic mixing matrix is proportional to $\operatorname{Tr}\left(Y_{u}^{1} Y_{u}^{2 \dagger}\right)$, we have taken in the scatter plot $\left|\left(Y_{u}^{2}\right)_{33} /\left(Y_{u}^{1}\right)_{33}\right| \leq 0.05$ (red points), $\left|\left(Y_{u}^{2}\right)_{33} /\left(Y_{u}^{1}\right)_{33}\right| \leq 0.15$ (green points) and $\left|\left(Y_{u}^{2}\right)_{33} /\left(Y_{u}^{1}\right)_{33}\right| \leq 0.3$ (blue points); the effects of the down quark Yukawa couplings have been neglected in this analysis, although their role is completely analogous. All the points in the plot reproduce the neutrino oscillation parameters within their experimental errors.

It is apparent from the plots that when the charged lepton Yukawa couplings have a negligible effect on the running (corresponding to $\left|\left(Y_{u}^{2}\right)_{33} /\left(Y_{u}^{1}\right)_{33}\right| \ll 1$ ), there is a fairly strong correlation between the radiatively generated $\theta_{13}$ and $\theta_{23}-\frac{\pi}{4}$. When the cut-off is $\Lambda=M_{\text {maj }}$, the numerical results are in good agreement with eq. (52), shown as a black solid line in the plot. In contrast, when $\Lambda=10^{18} \mathrm{GeV}$ there is a larger spread of the points, due to the additional RGE effects between $\Lambda$ and $M_{\text {maj. }}$. Besides, in this case the 
numerical results do not agree with eq. (52), since the RGE running between $\Lambda$ and $M_{\text {maj }}$ generates a non-vanishing (and negative) shift of $U_{23} / U_{33}$ at the scale $M_{\text {maj }}$, even if $U_{13}$ still vanishes. This produces, following eq. (50), the shift of the points to the left of the black solid line.

To summarize, from our analytical and numerical analysis, it follows that in the 2HDM extended with one right-handed neutrino it is generally expected a deviation of the atmospheric angle from the maximal value which is comparable to the reactor angle

$$
\left|\theta_{23}-\frac{\pi}{4}\right| \approx \theta_{13}
$$

unless the $\mathrm{CP}$ violating phase $\delta$ is very close to $\pi / 2$.

\section{Lepton flavour violation}

In the general 2HDM extended with RH neutrinos, one generically expects a misalignment in the charged lepton Yukawa couplings which will lead to new phenomena at low energies, apart from neutrino masses, in contrast to the standard see-saw scenario with just one Higgs doublet. This misalignment will generically arise already at tree level. However, even if the charged lepton Yukawa couplings are aligned at the cut-off scale $\Lambda$, radiative corrections from the neutrino Yukawa couplings from the RGE running above the Majorana mass scale will introduce off-diagonal entries in both charged lepton Yukawa matrices. Note that the radiative generation of neutrino masses requires a misalignment in the neutrino Yukawa couplings, hence some amount of flavour violation is necessarily generated via quantum corrections in the charged lepton sector.

To calculate the minimum amount of lepton flavour violation in the charged lepton sector, we assume that $Y_{e}^{1}, Y_{e}^{2}$ are diagonal at the cut-off scale $\Lambda>M_{\text {maj. }}$. Then, due to the radiative corrections from the neutrino Yukawa couplings $Y_{\nu}^{1}, Y_{\nu}^{2}$, both charged lepton Yukawa couplings become non-diagonal at the scale $M_{\text {maj }}$. As discussed in the previous section, we now redefine the charged lepton fields in order to bring the Yukawa coupling $Y_{e}^{1}$ into its diagonal form. As a result, the off-diagonal elements of the charged lepton Yukawa coupling read, at the Majorana mass scale:

$$
\begin{aligned}
& \left.\left(V_{e}^{L \dagger} Y_{e}^{2}\right)_{i j}\right|_{M_{\text {maj }}}=\frac{\log \frac{\Lambda}{M_{\text {maj }}}}{8 \pi^{2}}\left(-Y_{\nu}^{1} Y_{\nu}^{1 \dagger} Y_{e}^{2}-Y_{\nu}^{2} Y_{\nu}^{1 \dagger} Y_{e}^{2}\left(Y_{e}^{1}\right)^{-1} Y_{e}^{2}\right. \\
& \left.+Y_{\nu}^{1} Y_{\nu}^{2 \dagger} Y_{e}^{1}+Y_{\nu}^{2} Y_{\nu}^{2 \dagger} Y_{e}^{2}\right)_{i j} \\
& i<j \text {. }
\end{aligned}
$$

Below the Majorana mass scale the charged lepton Yukawa couplings are also affected by

the quantum effects, however the off-diagonal elements at low energies are still given by the previous expression, up to second order effects.

To estimate this contribution we assume $Y_{e}^{2}=\xi_{e} Y_{e}^{1}$, with at the cut-off scale. With this ansatz, eq. (54) reads:

$$
\left.\left(V_{e}^{L \dagger} Y_{e}^{2}\right)_{12}\right|_{M_{\mathrm{maj}}}=\frac{\log \frac{\Lambda}{M_{\mathrm{maj}}}}{8 \pi^{2}}\left(Y_{\nu}^{1}+\xi_{e} Y_{\nu}^{2}\right)_{1}\left(-\xi_{e} Y_{\nu}^{1 *}+Y_{\nu}^{2 *}\right)_{2} Y_{e 22}^{1} .
$$


Inserting this contribution into eq. (8) one obtains the approximate lower bound:

$$
\mathrm{BR}(\mu \rightarrow e \gamma) \gtrsim \frac{8 \alpha^{3}}{3 \pi^{3}}\left(\frac{\log \frac{\Lambda}{M_{\mathrm{maj}}}}{8 \pi^{2}}\right)^{2}\left|Y_{\nu 1}^{1}+\xi_{e} Y_{\nu 1}^{2}\right|^{2}\left|Y_{\nu 2}^{2}-\xi_{e}^{*} Y_{\nu 2}^{1}\right|^{2}\left|f\left(\frac{m_{t}^{2}}{m_{h}^{2}}\right) \frac{\left|\lambda_{6}\right| v^{2}}{m_{H}^{2}}\right|^{2}
$$

which is saturated when the charged lepton Yukawa couplings are aligned and when the Yukawa couplings of the heavy Higgs to the quarks are negligible.

To estimate the size of this lower bound, we will assume neutrino Yukawa couplings maximally misaligned with the form $Y_{\nu}^{1}=\frac{y_{1}}{\sqrt{2}}(0,1,1)^{T}, Y_{\nu}^{2}=\frac{y_{2}}{\sqrt{3}}(1,1,-1)^{T}$, being $y_{1}$ and $y_{2}$ the corresponding norms. With this choice, we obtain, in the limit $\left|\xi_{e}\right| \gg 1$ and $m_{H} \gg M_{Z}$ and taking $\Lambda=10^{4} M_{\mathrm{maj}}$,

$$
\mathrm{BR}(\mu \rightarrow e \gamma) \gtrsim 3 \times 10^{-15} \times\left|\lambda_{6} y_{1} y_{2} \xi_{e}^{2}\right|^{2}\left(\frac{m_{H}}{3 \mathrm{TeV}}\right)^{-4}
$$

Given that this bound is very conservative, the observation of the process $\mu \rightarrow e \gamma$ may be at the reach of the MEG experiment, which aims to $\mathrm{BR}(\mu \rightarrow e \gamma)>10^{-13}$ [27], provided the couplings are sizable and provided the extra scalar degrees of freedom are not too heavy.

\section{Conclusions}

We have considered in this paper an extension of the Standard Model by one extra Higgs doublet and one or more Majorana right-handed neutrinos, including in the Lagrangian all terms compatible with the Standard Model gauge symmetry. We have calculated, using a renormalization group approach, the quantum corrections to the neutrino parameters under the assumption that the right-handed Majorana mass scale is much larger than the mass of the Higgs mass eigenstates. We have argued that if the neutrino Yukawa couplings are misaligned, the radiatively generated contribution to the mass of the next-to-heaviest neutrino can be much larger than the tree level mass. Furthermore, for reasonable choices of the parameters of the model, the radiatively generated mass of the next-to-heaviest neutrino is a factor of a few smaller than the mass of the heaviest neutrino. Since the mass hierarchy depends only logarithmically on the masses of the extra degrees of freedom, this conclusion is fairly insensitive to the scales at which the new physics appears.

Hence, in this simple model two puzzles in neutrino physics can be simultaneously explained. First, the smallness of the neutrino masses is explained by the see-saw mecha-

nism. Secondly, the mild hierarchy between the atmospheric and the solar neutrino mass scales is explained by the radiative origin of the mass of the next-to-heaviest neutrino, which is suppressed by the loop factor but enhanced by the large logarithm of the ratio between the heavy Majorana mass scale and the heavy Higgs scale. Furthermore, by making the heavy Higgs scale sufficiently large, all the successes of the Standard Model can be preserved, since all low energy effects of the extended Higgs sector are suppressed at least by two powers of the heavy Higgs mass. 
The misalignment in the Yukawa couplings, necessary for the radiative generation of the solar neutrino mass scale, amounts to new sources of lepton flavour violation which also modify the structure of the leptonic mixing matrix through the renormalization group evolution. Therefore, we expect in this model deviations from the maximal atmospheric mixing and from a vanishing $\theta_{13}$ due to quantum effects. We have carefully calculated these corrections and we have found that the radiatively generated angle $\theta_{13}$ can be large enough to be measured in present and future experiments.

\section{Acknowledgements}

We are grateful to Carolin Bräuninger, Alberto Casas, Concha González García, Thomas Hambye, Alejandra Melfo and Enrico Nardi for useful discussions. This work was partially supported by the DFG cluster of excellence Origin and Structure of the Universe and by the Graduiertenkolleg Particle Physics at the Energy Frontier of New Phenomena.

\section{A Appendix}

\section{A.1 Quantum corrections below $M_{\text {maj }}$}

The one-loop $\beta$ functions of the multi-Higgs doublet model, including the dimension five operator which yields neutrino masses, have been derived in [28]. The $\beta$ functions of the charged lepton Yukawa couplings, $Y_{e}^{a}$, and the dimension five operators $\kappa^{a b}$ read, for energy scales below the right-handed neutrino Majorana mass scale,

$$
\begin{aligned}
\beta_{Y_{e}^{a}}= & \left(-\frac{9}{4} g^{2}-\frac{15}{4} g^{\prime 2}\right) Y_{e}^{a}+\left[3 \operatorname{Tr}\left(Y_{u}^{a \dagger} Y_{u}^{c}+Y_{d}^{a} Y_{d}^{c \dagger}\right)+\operatorname{Tr}\left(Y_{e}^{a} Y_{e}^{c \dagger}\right)\right] Y_{e}^{c} \\
& +Y_{e}^{a} Y_{e}^{c \dagger} Y_{e}^{c}+\frac{1}{2} Y_{e}^{c} Y_{e}^{c \dagger} Y_{e}^{a}, \\
\beta_{\kappa^{a b}}= & \frac{1}{2}\left[Y_{e}^{c} Y_{e}^{c \dagger} \kappa^{a b}+\kappa^{a b}\left(Y_{e}^{c} Y_{e}^{c \dagger}\right)^{T}\right]+2\left[Y_{e}^{c} Y_{e}^{b \dagger} \kappa^{a c}+\kappa^{c b}\left(Y_{e}^{c} Y_{e}^{a \dagger}\right)^{T}\right] \\
& -2\left[Y_{e}^{c} Y_{e}^{a \dagger}\left(\kappa^{c b}+\kappa^{b c}\right)+\left(\kappa^{a c}+\kappa^{c a}\right)\left(Y_{e}^{c} Y_{e}^{b \dagger}\right)^{T}\right] \\
& +\left[3 \operatorname{Tr}\left(Y_{u}^{a} Y_{u}^{c \dagger}+Y_{d}^{a \dagger} Y_{d}^{c}\right)+\operatorname{Tr}\left(Y_{e}^{a \dagger} Y_{e}^{c}\right)\right] \kappa^{c b} \\
& +\kappa^{a c}\left[3 \operatorname{Tr}\left(Y_{u}^{b} Y_{u}^{c \dagger}+Y_{d}^{b \dagger} Y_{d}^{c}\right)+\operatorname{Tr}\left(Y_{e}^{b \dagger} Y_{e}^{c}\right)\right] \\
& -3 g^{2} \kappa^{a b}+2 \lambda_{a c b d} \kappa^{c d},
\end{aligned}
$$

where summation over repeated indices is understood and the quartic couplings $\lambda$ are defined by $V \supset \frac{1}{2} \lambda_{a b c d}\left(\Phi_{a}^{\dagger} \Phi_{b}\right)\left(\Phi_{c}^{\dagger} \Phi_{d}\right)$.

In the case of only one right-handed neutrino and two Higgs doublets, $\kappa^{11}\left(m_{H}\right)$ can be approximately written at the leading log at any mass scale $m_{H}<M_{\text {maj }}$ in the form 
of eq. (18) which we repeat here for completeness:

$$
\begin{aligned}
\kappa^{11}\left(m_{H}\right) & \approx \kappa^{11}\left(M_{\text {maj }}\right)-\frac{1}{16 \pi^{2}} \beta_{\kappa^{11}}\left(M_{\text {maj }}\right) \log \frac{M_{\text {maj }}}{m_{H}} \\
& \equiv \kappa^{11}\left(M_{\text {maj }}\right)+B_{1 a} \kappa^{a 1}+\kappa^{1 a} B_{1 a}^{T}+b \kappa^{22}
\end{aligned}
$$

where we have defined flavour matrices $B_{11}, B_{12}$ and the complex number $b$ by

$$
\begin{aligned}
\frac{16 \pi^{2}}{\log \frac{M_{\text {maj }}}{m_{H}}} B_{11} & =-\frac{1}{2} Y_{e}^{2} Y_{e}^{2 \dagger}+\frac{3}{2} Y_{e}^{1} Y_{e}^{1 \dagger}-3 \operatorname{Tr}\left(Y_{u}^{1} Y_{u}^{1 \dagger}+Y_{d}^{1 \dagger} Y_{d}^{1}\right)-\operatorname{Tr}\left(Y_{e}^{1 \dagger} Y_{e}^{1}\right)+\frac{3}{2} g^{2}-\lambda_{1} \\
\frac{16 \pi^{2}}{\log \frac{M_{\text {maj }}}{m_{H}}} B_{12} & =2 Y_{e}^{2} Y_{e}^{1 \dagger}-3 \operatorname{Tr}\left(Y_{u}^{1} Y_{u}^{2 \dagger}+Y_{d}^{1 \dagger} Y_{d}^{2}\right)-\operatorname{Tr}\left(Y_{e}^{1 \dagger} Y_{e}^{2}\right)-2 \lambda_{6} \\
\frac{16 \pi^{2}}{\log \frac{M_{\text {maj }}}{m_{H}}} b & =-2 \lambda_{5}
\end{aligned}
$$

\section{A.2 Quantum corrections above $M_{\text {maj }}$}

In the case that the cut-off scale of the theory, $\Lambda$, is larger than $M_{\text {maj }}$, the relevant matricial couplings of the leptonic Lagrangian are the charged lepton Yukawa couplings, $Y_{e}^{a}$, the neutrino Yukawa couplings, $Y_{\nu}^{a}$, and the right-handed Majorana mass matrix $M_{\mathrm{M}}$. The corresponding $\beta$-functions are:

$$
\begin{aligned}
\beta_{Y_{e}^{a}}^{\Lambda}= & \beta_{Y_{e}^{a}}+\operatorname{Tr}\left(Y_{\nu}^{a \dagger} Y_{\nu}^{c}\right) Y_{e}^{c}-2 Y_{\nu}^{c} Y_{\nu}^{a \dagger} Y_{e}^{c}+\frac{1}{2} Y_{\nu}^{c} Y_{\nu}^{c \dagger} Y_{e}^{a}, \\
\beta_{Y_{\nu}^{a}}^{\Lambda}= & {\left[-\frac{9}{4} g^{2}-\frac{3}{4} g^{\prime 2}\right] Y_{\nu}^{a}+\left[3 \operatorname{Tr}\left(Y_{u}^{a} Y_{u}^{c \dagger}+Y_{d}^{a \dagger} Y_{d}^{c}\right)+\operatorname{Tr}\left(Y_{\nu}^{a} Y_{\nu}^{c \dagger}+Y_{e}^{a \dagger} Y_{e}^{c}\right)\right] Y_{\nu}^{c} } \\
& -2 Y_{e}^{c} Y_{e}^{a \dagger} Y_{\nu}^{c}+Y_{\nu}^{a} Y_{\nu}^{c \dagger} Y_{\nu}^{c}+\frac{1}{2} Y_{e}^{c} Y_{e}^{c \dagger} Y_{\nu}^{a}+\frac{1}{2} Y_{\nu}^{c} Y_{\nu}^{c \dagger} Y_{\nu}^{a}, \\
\gamma_{M_{\mathrm{M}}}^{\Lambda}= & -M_{\mathrm{M}}^{-1}\left[\left(Y_{\nu}^{c \dagger} Y_{\nu}^{c}\right)^{T} M_{\mathrm{M}}+M_{\mathrm{M}} Y_{\nu}^{c \dagger} Y_{\nu}^{c}\right] .
\end{aligned}
$$

The running above $M_{\text {maj }}$ modifies some of the expressions that we have derived in this

paper. The effects of the running can be easily incorporated in our results by substituting

$$
B_{1 a} \rightarrow B_{1 a}+B_{1 a}^{\Lambda}, \quad \kappa^{a b} \rightarrow \frac{Y_{\nu}^{a} Y_{\nu}^{b T}}{M_{\text {maj }}} .
$$

More concretely, the values for $B_{1 a}^{\Lambda}$ read:

$$
\begin{aligned}
\frac{16 \pi^{2}}{\log \frac{\Lambda}{M_{\mathrm{maj}}}} B_{11}^{\Lambda}= & \frac{9}{4} g^{2}+\frac{3}{4} g^{\prime 2}-3 \operatorname{Tr}\left(Y_{u}^{1} Y_{u}^{1 \dagger}+Y_{d}^{1 \dagger} Y_{d}^{1}\right)-\operatorname{Tr}\left(Y_{\nu}^{1} Y_{\nu}^{1 \dagger}+Y_{e}^{1 \dagger} Y_{e}^{1}\right) \\
& -\frac{1}{2} Y_{e}^{2} Y_{e}^{2 \dagger}+\frac{3}{2} Y_{e}^{1} Y_{e}^{1 \dagger}-\frac{1}{2} Y_{\nu}^{1} Y_{\nu}^{1 \dagger}-\frac{1}{2} Y_{\nu}^{2} Y_{\nu}^{2 \dagger}, \\
\frac{16 \pi^{2}}{\log \frac{\Lambda}{M_{\mathrm{maj}}}} B_{12}^{\Lambda}= & 2 Y_{e}^{2} Y_{e}^{1 \dagger}-3 \operatorname{Tr}\left(Y_{u}^{1} Y_{u}^{2 \dagger}+Y_{d}^{1 \dagger} Y_{d}^{2}\right)-\operatorname{Tr}\left(Y_{e}^{1 \dagger} Y_{e}^{2}+Y_{\nu}^{1} Y_{\nu}^{2 \dagger}\right) .
\end{aligned}
$$


Note that the running above $M_{\text {maj }}$ does not modify the value of $b$ nor $m_{2}$, cf. eq. (27).

Besides, the running above $M_{\text {maj }}$ also affects the structure of $V_{e}^{L}$ at low energies. This can be taken into account with the following substitution in the relevant formulas:

$$
\beta_{Y_{e}^{1}} \log \frac{M_{\text {maj }}}{m_{H}} \rightarrow \beta_{Y_{e}^{1}} \log \frac{M_{\text {maj }}}{m_{H}}+\beta_{Y_{e}^{1}}^{\Lambda} \log \frac{\Lambda}{M_{\text {maj }}} .
$$

\section{References}

[1] For a review, see M. C. Gonzalez-Garcia, M. Maltoni, Phys. Rept. 460 (2008) 1-129.

[2] M. Maltoni, T. Schwetz, PoS IDM2008 (2008) 072.

[3] P. Minkowski, Phys. Lett. B 67 (1977) 421; M. Gell-Mann, P. Ramond and R. Slansky, Proceedings of the Supergravity Stony Brook Workshop, New York 1979, eds. P. Van Nieuwenhuizen and D. Freedman; T. Yanagida, Proceedinds of the Workshop on Unified Theories and Baryon Number in the Universe, Tsukuba, Japan 1979, eds. A. Sawada and A. Sugamoto; R. N. Mohapatra, G. Senjanovic, Phys.Rev.Lett. 44 (1980)912, ibid. Phys.Rev. D23 (1981) 165; S. L. Glashow, The Future Of Elementary Particle Physics, In ${ }^{*}$ Cargese 1979, Proceedings, Quarks and Leptons*, 687-713 and Harvard Univ.Cambridge - HUTP-79-A059 (79,REC.DEC.) 40p. J. Schechter and J. W. F. Valle, Phys. Rev. D 22 (1980) 2227.

[4] J. A. Casas, A. Ibarra and F. Jimenez-Alburquerque, JHEP 0704 (2007) 064.

[5] E. Ma, Phys. Rev. D73, 077301 (2006).

[6] See, for example, J. Kubo, E. Ma, D. Suematsu, Phys. Lett. B642 (2006) 18-23; C. Boehm, Y. Farzan, T. Hambye, S. Palomares-Ruiz, S. Pascoli, Phys. Rev. D77 (2008) 043516; M. Aoki, S. Kanemura, O. Seto, Phys. Rev. Lett. 102 (2009) 051805, Phys. Rev. D80 (2009) 033007; J. Kubo, D. Suematsu, Phys. Lett. B643 (2006) 336-341; N. Haba, K. Tsumura, JHEP 1106 (2011) 068.

[7] W. Grimus, H. Neufeld, Nucl. Phys. B325 (1989) 18. W. Grimus and L. Lavoura, Phys. Lett. B 546 (2002) 86.

[8] W. Grimus, H. Neufeld, Phys. Lett. B486 (2000) 385-390.

[9] T. D. Lee, Phys. Rev. D8, 1226-1239 (1973).

[10] For a recent review, see G. C. Branco, P. M. Ferreira, L. Lavoura, M. N. Rebelo, M. Sher, J. P. Silva, arXiv:1106.0034 [hep-ph]].

[11] M. E. Peskin, T. Takeuchi, Phys. Rev. Lett. 65 (1990) 964-967.

[12] J. F. Gunion, H. E. Haber, Phys. Rev. D67 (2003) 075019. 
[13] H. E. Haber, D. O'Neil, Phys. Rev. D83 (2011) 055017.

[14] G. D'Ambrosio, G. F. Giudice, G. Isidori and A. Strumia, Nucl. Phys. B 645, 155 (2002); A. Pich and P. Tuzon, Phys. Rev. D 80, 091702 (2009); A. J. Buras, M. V. Carlucci, S. Gori and G. Isidori, JHEP 1010, 009 (2010); C. B. Braeuninger, A. Ibarra and C. Simonetto, Phys. Lett. B 692 (2010) 189; M. Jung, A. Pich and P. Tuzon, JHEP 1011 (2010) 003, Phys. Rev. D 83 (2011) 074011.

[15] M. L. Brooks et al. [MEGA Collaboration], Phys. Rev. Lett. 83 (1999) 1521.

[16] P. Paradisi, JHEP 0602 (2006) 050.

[17] J. Hisano, S. Sugiyama, M. Yamanaka et al., Phys. Lett. B694 (2011) 380-385.

[18] J. Laiho, E. Lunghi and R. S. Van de Water, Phys. Rev. D 81 (2010) 034503.

[19] K. Nakamura et al. [ Particle Data Group Collaboration ], J. Phys. G G37 (2010) 075021.

[20] A. J. Buras, S. Jager, J. Urban, Nucl. Phys. B605 (2001) 600-624.

[21] E. Lunghi and A. Soni, JHEP 0709 (2007) 053. See also A. Lenz, U. Nierste, JHEP 0706 (2007) 072.

[22] J. A. Casas, J. R. Espinosa, A. Ibarra, I. Navarro, Nucl. Phys. B573 (2000) 652684, Nucl. Phys. B569 (2000) 82-106; J. R. Ellis, S. Lola, Phys. Lett. B458 (1999) 310-321; P. H. Chankowski, W. Krolikowski, S. Pokorski, Phys. Lett. B473 (2000) 109-117; S. Antusch, J. Kersten, M. Lindner, M. Ratz, Nucl. Phys. B674 (2003) 401-433.

[23] S. T. Petcov, S. T. Toshev, Phys. Lett. B143 (1984) 175; K. S. Babu, E. Ma, Phys. Rev. Lett. 61 (1988) 674; E. Ma, Phys. Lett. B456 (1999) 48-53.

[24] L. J. Hall, H. Murayama, N. Weiner, Phys. Rev. Lett. 84 (2000) 2572-2575.

[25] J. A. Casas and A. Ibarra, Nucl. Phys. B 618 (2001) 171. A. Ibarra, G. G. Ross, Phys. Lett. B591 (2004) 285-296.

[26] A. Ibarra, E. Molinaro, S. T. Petcov, arXiv:1103.6217 [hep-ph]].

[27] A. Maki, AIP Conf. Proc. 981 (2008) 363-365.

[28] W. Grimus, L. Lavoura, Eur. Phys. J. C39 (2005) 219-227. 